Review

\title{
Structural Diversity and Function of Xyloglucan Sidechain Substituents
}

\author{
Alex Schultink ${ }^{1}$, Lifeng Liu ${ }^{2}$, Lei Zhu ${ }^{2}$ and Markus Pauly 1,2,* \\ 1 Department of Plant and Microbial Biology, University of California, Berkeley, CA 94720, USA; \\ E-Mail: aschult@berkeley.edu \\ 2 Energy Biosciences Institute, University of California, Berkeley, CA 94720, USA; \\ E-Mails: lifeng.liu@ berkeley.edu (L.L.); lei.zhu@berkeley.edu (L.Z.) \\ * Author to whom correspondence should be addressed; E-Mail: mpauly69@berkeley.edu; \\ Tel.: +1-510-642-1722.
}

External Editor: Philip Harris

Received: 7 October 2014; in revised form: 3 November 2014 / Accepted: 4 November 2014 /

Published: 13 November 2014

\begin{abstract}
Xyloglucan (XyG) is a hemicellulose found in the cell walls of all land plants including early-divergent groups such as liverworts, hornworts and mosses. The basic structure of XyG, a xylosylated glucan, is similar in all of these plants but additional substituents can vary depending on plant family, tissue, and developmental stage. A comprehensive list of known $\mathrm{XyG}$ sidechain substituents is assembled including their occurrence within plant families, thereby providing insight into the evolutionary origin of the various sidechains. Recent advances in DNA sequencing have enabled comparative genomics approaches for the identification of $\mathrm{XyG}$ biosynthetic enzymes in Arabidopsis thaliana as well as in non-model plant species. Characterization of these biosynthetic genes not only allows the determination of their substrate specificity but also provides insights into the function of the various substituents in plant growth and development.
\end{abstract}

Keywords: xyloglucan; hemicellulose; plant cell walls; polysaccharide; glycosyltransferase 


\section{Introduction}

The cells of plants are encased in a wall, a sophisticated composite material that is composed of the polysaccharides cellulose, various non-cellulosic polysaccharides including hemicelluloses and pectic polysaccharides, structural glycoproteins and, in mature secondary walls, the polyphenol lignin [1,2]. One of the hemicelluloses present in the walls of all land plants (embryophytes) is xyloglucan (XyG). $\mathrm{XyG}$ is the most abundant hemicellulose in the primary walls, i.e., the walls of elongating cells [1], of many plants and can also occur as a storage amyloid in seeds [3].

In primary walls, XyG cross-links cellulose microfibrils [4], forming a strong but extensible XyG-cellulose network [2,5-8]. XyG may act as a spacer-molecule preventing aggregation of cellulose microfibrils $[9,10]$ or a mediator-molecule enabling interactions between cellulose microfibrils and other wall matrix polymers $[11,12]$. Once deposited into the wall, XyG can undergo metabolism and turnover, which is thought to play a role in cell elongation [13]. Despite its prominence in models of the primary cell wall, an Arabidopsis mutant lacking detectable XyG has surprisingly only minor growth phenotypes suggesting that XyG is not as critical for wall architecture and function as once thought [14]. Indeed, recent data from mechanical testing experiments on primary walls using XyG modifying enzymes point towards a more limited but nevertheless significant structural role of $\mathrm{XyG}$ in wall mechanics [15].

In some plant species including tamarind (Tamarindus indica), nasturtium (Tropaeolum majus) and Hymenaea courbaril, XyG functions as a seed storage polymer [3,16,17]. In these species, XyG is deposited in large quantities during the development of the seed and upon germination is degraded to provide energy for the emerging seedling [18].

$\mathrm{XyG}$ is used in various food, industrial and pharmaceutical applications [19]. Seed storage XyG is often used for these purposes as it is easier to extract than $\mathrm{XyG}$ embedded in the wall matrix of cells in vegetative organs and is highly abundant in certain species. XyG isolated from tamarind seed has been tested successfully as a carrier for specific drug delivery systems [20,21] and the administration of antibiotics and treatment of ulcers [22]. Chemical derivatives of XyG oligosaccharides can also be used as biodegradable surfactants [23] and to activate cellulosic surfaces with a variety of functional groups [24].

\section{XyG Structural Nomenclature and Presently Known Sidechain Diversity}

XyG consists of a $\beta-1,4$ glucan chain that is decorated with a certain pattern of glycosyl groups $[25,26]$. The identity and position of the glycosyl units attached to a particular glucosyl backbone residue can be diverse and a one letter code for the various sidechains has been established, which allows for a concise depiction of specific XyG structures [27]. In this system, a backbone glucosyl residue without any further substitution is depicted by the letter $\mathrm{G}$ and a backbone glucosyl residue with an additional xylosyl unit at the O-6 position is described as X. X groups can carry additional glycosyl-moieties and so far 17 different sidechain structures have been identified, each with a unique one-letter abbreviation (Figure 1) [28]. The glycosyl units found in these various sidechains include D-galactose, D-xylose, L-arabinopyranose, L-arabinofuranose, D-galacturonic acid, L-fucose, and L-galactose. 
Figure 1. XyG sidechain composition and diversity. A one letter nomenclature has been developed for all XyG sidechains identified to date [27,28]. The backbone $\beta$-(1,4)-glucosyl residues are identified in black. Acetylated sidechains are indicated by an underline, as in $\underline{L}$ or $\underline{S}$. The directionality of the glycosidic bonds is indicated by an arrow, with the number referring to the hydroxyl-group of the sugar (arrow-head) that is linked to the anomeric carbon (C1) of the other sugar. Similar oligosaccharide structures are boxed.

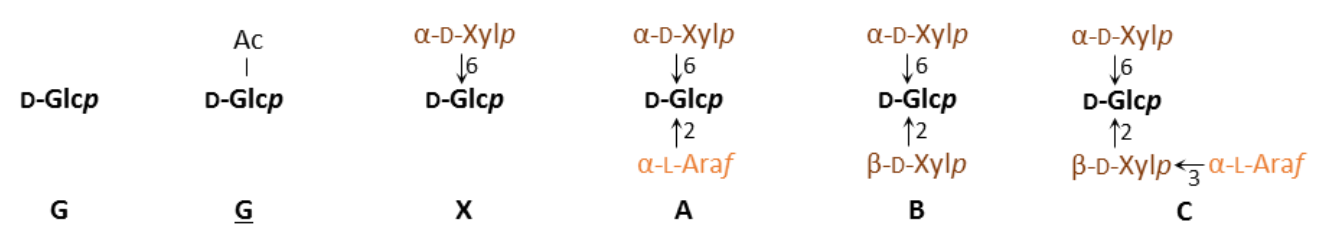

\begin{tabular}{|c|c|c|c|c|c|c|}
\hline $\begin{array}{c}\beta \text {-D-Galp } \\
\downarrow 2 \\
\alpha \text {-D-Xylp } \\
\downarrow 6 \\
\text { D-Glcp }\end{array}$ & $\begin{array}{c}\text { AC } \\
\mid \\
\beta-D-G a l p \\
\downarrow^{2} \\
\alpha-D-X y l p \\
\downarrow 6 \\
\text { D-Glcp }\end{array}$ & $\begin{array}{c}\alpha \text {-L-Arap } \\
\downarrow^{2} \\
\alpha \text {-D-Xylp } \\
\downarrow 6 \\
\text { D-Glcp }\end{array}$ & $\begin{array}{c}\beta \text {-D-GalAp } \\
\downarrow^{2} \\
\alpha \text {-D-Xylp } \\
\downarrow 6 \\
\text { D-Glcp }\end{array}$ & $\begin{array}{c}\beta \text {-D-Xylp } \\
\downarrow 2 \\
\alpha \text {-D-Xylp } \\
\downarrow 6 \\
\text { D-Glcp }\end{array}$ & $\begin{array}{c}\alpha \text {-L-Araf } \\
\downarrow 2 \\
\alpha \text {-D-Xylp } \\
\downarrow 6 \\
\text { D-Glcp }\end{array}$ & $\begin{array}{c}\text { Ac } \\
\mid \\
\alpha-\text {-Araf } \\
\downarrow^{2} \\
\alpha-D-X y l p \\
\downarrow 6 \\
\text { D-Glcp }\end{array}$ \\
\hline $\mathbf{L}$ & $\underline{\mathbf{L}}$ & D & $\mathbf{Y}$ & $\mathbf{U}$ & $\mathbf{S}$ & $\underline{\mathbf{S}}$ \\
\hline
\end{tabular}

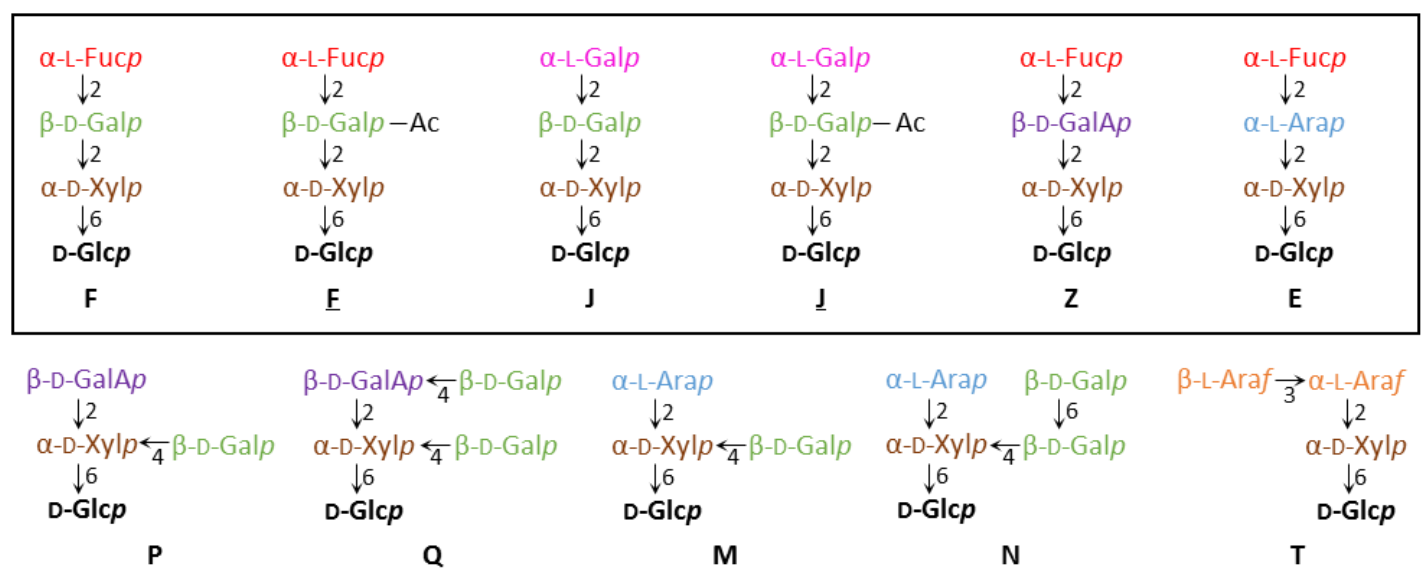

XyG sidechains can be placed into groups based on similarities in structure. For example, the L, S, $\mathrm{D}, \mathrm{Y}$ and $\mathrm{U}$ units differ only in the identity of the terminal glycosyl group linked to the O-2 position of the xylosyl-residue (D-galactose, L-arabinofuranose, L-arabinopyranose, D-galacturonic acid, and D-xylose, respectively). A similar grouping can be composed of the F, E, J and Z sidechains, which contain terminal $\alpha$-(1,2)-L-fucosyl-residues or the conformationally analogous L-galactosyl-residue on the L, D, and Y units.

$\mathrm{XyG}$ sidechains can be further modified by the addition of one or multiple acetyl groups on specific glycosyl groups $[29,30]$. Acetylated sidechains are depicted with an underline, such as in $\underline{S}$ and $\underline{L}$. To date only three distinct XyG glycosyl units have been found to be acetylated. These include unsubstituted glucosyl backbone residues $(\underline{G})$ [31,32], the arabinofuranosyl-residue of $\underline{S}$ groups [33], and the D-galactosyl-residue of $\underline{\mathrm{L}}, \underline{\mathrm{F}}$ and $\underline{\mathrm{J}}$ groups $[29,30,34]$. Acetyl groups have been shown to migrate between hydroxyl-groups within a particular glycosyl unit to reach a distribution equilibrium [35-37]. Some plant species including pea (Pisum sativum) have been shown to have two or more acetyl-substituents on a single galactosyl-residue [38]. A distinction between singly and doubly acetylated sidechains is not made under the current nomenclature system. NMR evidence has suggested the existence of methylated XyG sidechains in certain species, however specific structures of these groups have not been elucidated [39]. 


\section{XyG Biodiversity}

Although antibodies have indicated the presence of epitopes that are recognized by XyG antibodies in the walls of aquatic green algae from the Charophyta [40] and these algae possess homologs of genes known to be involved in XyG biosynthesis [41], specific XyG structures have hitherto only been characterized in land plants (embryophytes) (Figures 2 and 3). Besides the defining X units, further sidechain extension by a galactosyl-moiety is a very common feature and seems to occur in all plant species suggesting an important functional role. The fucosyl-residue seems to first appear in the hornworts and is a common feature in nearly all vascular plants investigated. Interestingly, a small group of eudicots including the Lamiales, which include basil (Ocimum basilicum), olive (Olea europaea), and plantain (Plantago major), the Solanales, which include tomato (Solanum lycopersicum), potato (Solanum tuberosum), and tobacco (Nicotiana tabacum), do not contain fucosylated XyG in their leaf tissue [42]. However, fucosylated XyG has been found in the pollen tube of Nicotiana alata [43] indicating that this $\mathrm{XyG}$ structure has been retained in some reproductive tissues and hinting at a functional significance in tip-growing tissues.

Figure 2. XyG structures of non-eudicot land plants. In each order or clade, XyG xylosylation motifs and sidechains are summarized from that reported for individual species within the group. See Table $\mathrm{S} 1$ for a detailed listing with plant families and species names and Figure 3 for the XyG structures of eudicots. The phylogenetic tree is adapted and modified from Hsieh and Harris [44] and that proposed by the Angiosperm Phylogeny Group III [45].

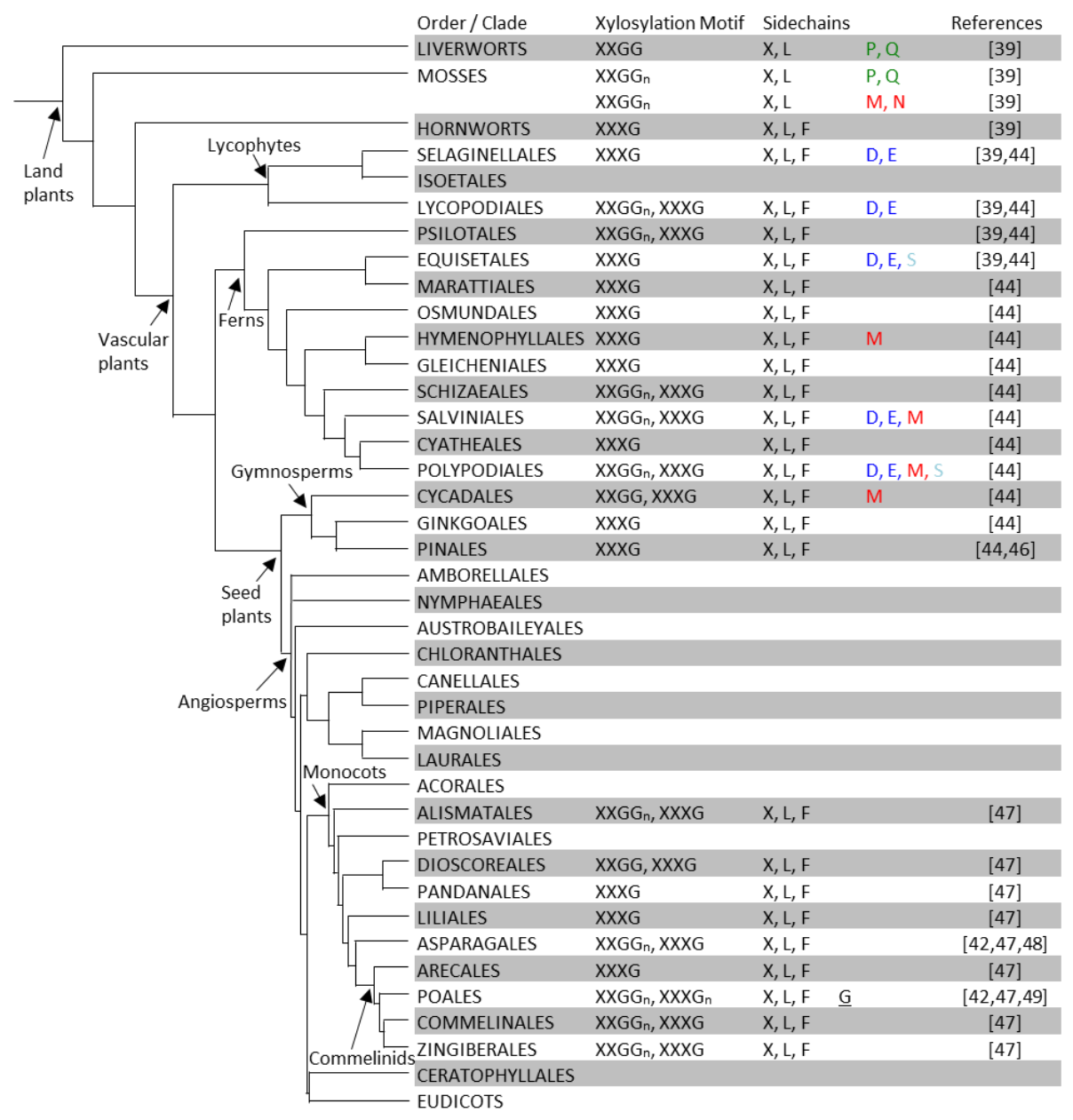


Figure 3. XyG structures of eudicot plants. In each order or family, XyG xylosation motifs structures and sidechains are summarized from individual species or tissue types. Two rows for a plant order indicates that different XyG structures were found in different tissues of a species of this order. For details see Table S1. The phylogenetic tree is adapted and modified from that proposed by the Angiosperm Phylogeny Group III [45].

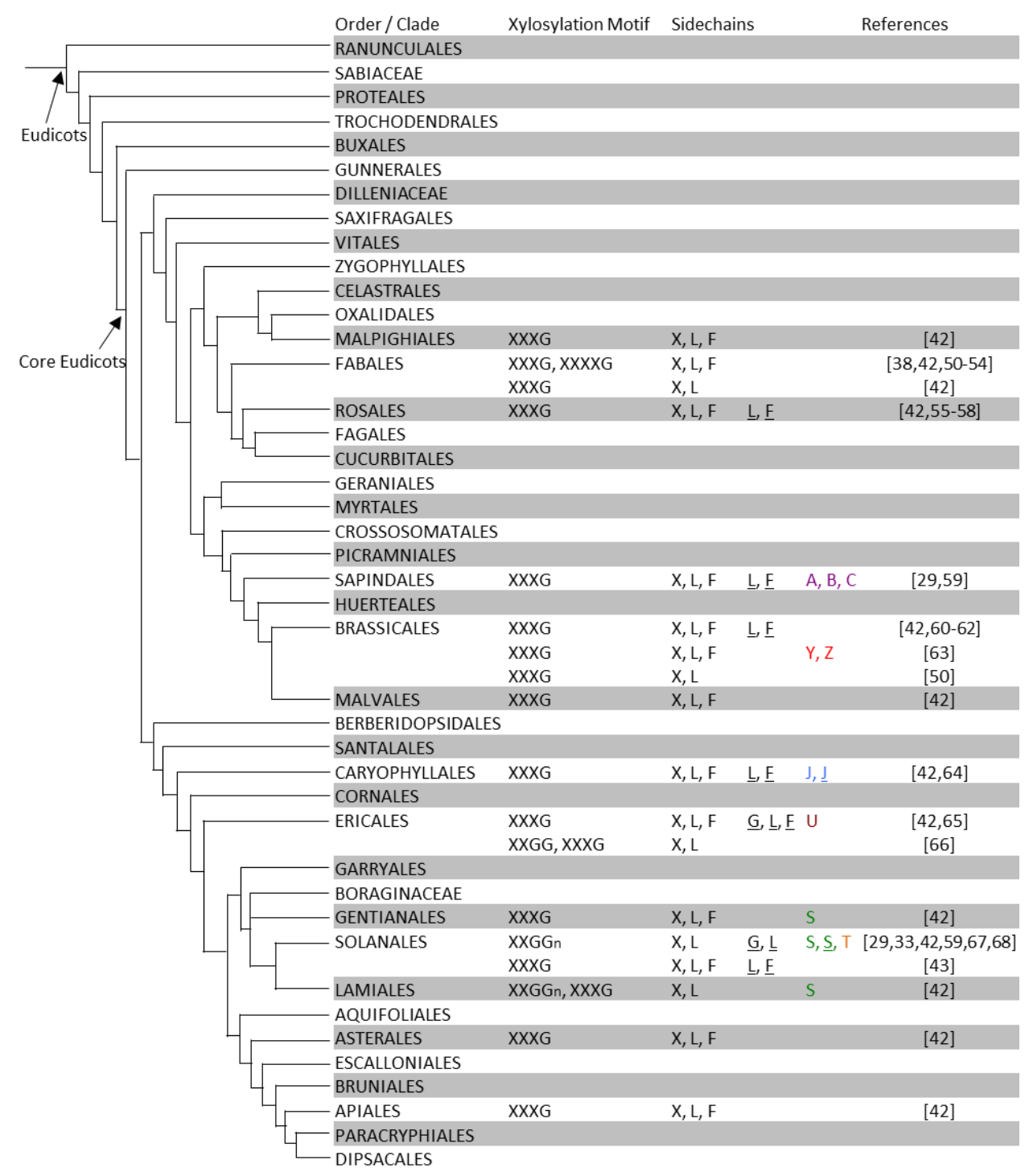

Unlike $\mathrm{X}, \mathrm{L}$ and $\mathrm{F}$, which are found in many or all clades throughout the land plant phylogeny, the occurrence of some other sidechains appears restricted to one or a few clades. For example, the P and Q sidechains have so far only been found in the liverwort and moss clades [39]. The P and Q sidechains are also unique as they contain branched sidechains with galactosyl-units bound to the xylosyl-residues at the O-4 position. This branching moiety is also present in the $\mathrm{M}$ and $\mathrm{N}$ sidechains of $\mathrm{XyG}$ found in the moss Physcomitrella patens [39]. Several species of ferns and a cycad also contain the M sidechain, a moiety that has not been reported to be present in any angiosperm species to date $[39,44]$. Sidechains containing arabinopyranosyl-residues ( $\mathrm{D}$ and $\mathrm{E}$ ) have been found in seedless vascular plants including lycophytes and ferns $[39,44]$, but not in seed plants. The $S$ sidechain containing arabinofuranosyl-residues has been found in the ferns (Equisetales and Polypodiales) [39], but is lacking in higher plant orders including the gymnosperms, monocots, and most of the eudicots with the exception of the subgroup of the Gentianales, 
Lamiales, and Solanales. The phylogenetic distance between this subgroup and the seedless plants suggests that the glycosyltransferases that lead to XyG arabinofuranosylation have evolved independently at least twice [69].

The $\mathbf{J}$ sidechain contains an L-galactosyl-residue instead of the conformationally analogous fucosylresidue found in $\mathrm{F}$ sidechains. $\mathrm{XyG}$ containing the $\mathrm{J}$ sidechain has been identified in jojoba seeds (Simmondsia chinensis) [70]. In suspension cultures of sycamore (Acer pseudoplatanus) the sidechains A, $\mathrm{B}$, and $\mathrm{C}$ were found [29,59], each of which contain an additional sugar residue on the glucosyl backbone at the $\mathrm{O}-2$ position.

Another feature of XyG is the $O$-acetyl-moiety. $O$-acetyl-substituents have only rarely been reported as this substituent is lost when $\mathrm{XyG}$ is extracted with alkali, a common method for $\mathrm{XyG}$ extraction from wall materials [71]. The function of the acetyl-substituent on XyG is not well understood but may influence polymer solubility as well as enzymatic accessibility. Removal of $O$-acetyl-substituents on $\mathrm{XyG}$ from the galactosyl-sidechains does not lead to any change in the binding capacity to cellulose [37].

$\mathrm{XyG}$ sidechains are not randomly positioned but are organized into $\mathrm{XyG}$ oligosaccharide motifs. These oligosaccharide motifs can be observed when XyG is digested with endoglucanases [72], which cleave XyG at unsubstituted glucosyl-residues. The oligosaccharides released by digestion of the XyG of Arabidopsis and many other dicot species share the common xylosylation motif of "XXXG", where the backbone largely consists of three xylosylated (and possibly further glycosylated) glucosyl units followed by one unsubstituted glucosyl residue [73]. A reduction in XyG backbone xylosylation is observed in the Poaceae, such as barley (Hordeum vulgare) and rice (Oryza sativa), which harbor a xylosylation motif of "XXGGn" with n typically ranging from one to three [42]. A decreased degree of xylosylation is also observed in the Solanaceae, such as tobacco and tomato [42,67], which have "XXGG" as the dominant XyG xylosylation motif (Figures 2 and 3, Table S1). In plants with the "XXGGn" motif $O$-acetylation of the glucan backbone appears to occur as an alternative to xylosylation at some positions. Additional xylosylation motifs have also been identified including XXXXG and $\mathrm{XXXXXG}$ as part of seed storage $\mathrm{XyG}$ [50]. While the majority of XyG oligosaccharides released from a particular species and tissue follow the dominant xylosylation pattern, there are exceptions. For example, $\mathrm{XyG}$ digestions of both monocot and dicot species including Arabidopsis were found to contain minor amounts of the XXG oligosaccharide [47,74]. These structures may result from partial degradation and reincorporation of $\mathrm{XyG}$ oligosaccharides within the wall [75].

The structure of $\mathrm{XyG}$ can vary within a single plant species depending on the tissue, cell type, and even position within the wall. One of the most prominent examples is represented by seed XyG. The storage XyG of several species including nasturtium and tamarind does not harbor fucosyl- or acetyl-groups, whereas these structures are present in the XyG found in the primary wall of these species [50]. Seed cotyledon parenchyma cells from these plants produce both types of $\mathrm{XyG}$, which are deposited in different layers of the wall [76].

Other specialized tissues have also been reported to have $\mathrm{XyG}$ structures differing from the most prevalent form of XyG in a particular species. The Y sidechain, containing a charged galacturonic acid, was identified from Arabidopsis root tissue and appears to be present only in root hairs [63]. In the Solanaceae, where the dominant XyG xylosylation motif is XXGG and fucosylation is typically not observed, fucosylated XXXG-type XyG has been observed specifically in pollen tubes [43]. Cell-specific differences in XyG fucosylation have also been observed via antibody labeling in certain species within the Poaceae, in which phloem cells appear to have fucosylated XyG whereas neighboring ones do not [77]. 
While the above examples represent qualitative differences in XyG structure within a plant, quantitative differences in the relative abundance of specific XyG sidechains and oligosaccharide motifs are also common. For example, in pea XLFG oligosaccharide motifs make up approximately $25 \%$ of the XyG in leaves whereas they only account for $5 \%$ of the XyG in etiolated stems [38].

\section{XyG Biosynthesis}

Numerous proteins involved in XyG biosynthesis have been identified (Figure 4) [26]. All these proteins have been shown to be localized in the Golgi, where XyG is synthesized [78,79]. The XyG glucan backbone is believed to be synthesized by proteins of the cellulose synthase-like C (CSLC) family. The TmCSLC4 gene is highly expressed in nasturtium (Tropaeolum majus) seeds, which contain a large abundance of galactosylated XyG. Heterologous expression of TmCSLC4 and its ortholog AtCSLC4 in Pichia pastoris resulted in the production of $\beta-(1,4)$ glucan in vitro [80]. Proteins from glycosyltransferase (GT) family 34 have been shown to act as XyG xylosyltransferases (XXT) [81-83]. Of the five XXTs in Arabidopsis, XXT1, XXT2 and XXT5 have the most significant effect on XyG biosynthesis when knocked out [14,84-86]. A double $x x t 1$ xxt2 knockout mutant lacked detectable XyG and exhibited abnormal root hairs and slow growth compared to wild type plants $[14,86]$. Overexpression of AtXXT3 could partially restore $\mathrm{XyG}$ abundance in $x x t 2, x x t 5$ and $x x t 2 x x t 5$ mutants indicating it is likely to represent a XyG xylosyltransferase [85]. Heterologously expressed AtXXT4 showed the ability to transfer UDP-xylose onto cellohexaose, as previously shown for AtXXT1 and AtXXT2, indicating it is also likely to be xylosyltransferase involved in XyG biosynthesis [85]. Recently, a putative rice XXT (OsXXT1) restored XyG structures and eliminated root hair defects when expressed in the Arabidopsis $x x t 1 \quad x x t 2$ mutant indicating that OsXXT1 has the same activity as its Arabidopsis XXT orthologs [87]. In the Solanaceae and Poaceae, the glucan backbone of XyG is $O$-acetylated $(\underline{\mathrm{G}})$ and has a lower degree of xylosylation $\left(\mathrm{XXGG}_{\mathrm{n}}\right.$ type $\mathrm{XyG}$; Figure 4). A XyG backbone $O$-acetyltransferase has not been identified to date and the mechanism of the reduction in XyG backbone xylosylation remains elusive.

The xylosyl substituents are often additionally glycosylated, most commonly with a galactosyl-residue. MURUS 3 (MUR3) from the GT family 47 exhibits XyG $\beta$ - $(1,2)$-galactosyltransferase activity and is responsible for galactosylation of the third xylosyl residue of the XXXG XyG oligosaccharide motif (i.e., the product is XXLG) [88] while Xyloglucan L-sidechain Galactosyltransferase Position 2 (XLT2), another GT47 galactosyltransferase, seems to be specific for galactosylating the second xylosyl residue (i.e., XXXG will be converted to XLXG) based on genetic experiments in Arabidopsis [89]. These enzymes thus exhibit regio-selectivity in that they add the same glycosyl-residue to the same glycosylacceptor, but the acceptor-moiety has to be in a specific structural environment on the XyG. The determinant of the GT protein structure for this regio-selectivity is not known and it will be interesting to crystalize these proteins to discover the mechanism and specific recognition motifs. Regio-selectivity cannot be excluded for other XyG GTs such as the xylosyltransferases. The double mutant xlt2 mur3 lacks substitution of the xylosyl-residues thus harboring a XyG made nearly entirely of XXXG units and resulting in a dwarfed phenotype in Arabidopsis [89]. MUR3 orthologs from other species such as Eucalyptus grandis and Solanum lycopersicum restore the galactosylated $\mathrm{XyG}$ when expressed in the mur3.1 or $x l t 2$ mur3 Arabidopsis mutants, suggesting that they exhibit the same activity as AtMUR3 [69,90]. Two GT47 homologs of AtXLT2 in tomato were found to result in the production of arabinosylated XyG 
when expressed in Arabidopsis, suggesting that both proteins, named Xyloglucan S-sidechain Transferase (XST) 1 and 2, represent arabinofuranosyltransferases and lead to the synthesis of the S sidechain found in tomato [69]. Overexpressing XST1 or XST2 in the xlt2 mur3 double mutant rescued the dwarfed mutant phenotype indicating an equivalent function in plant growth for the $\mathrm{L}$ and $\mathrm{S}$ sidechains in this case [69]. A root hair specific gene, Xyloglucan-specific Galacturonosyltransferase1 (XUT1), was found to be required for the presence of galacturonic acid (Y sidechains) on XyG in Arabidopsis. Knock-out of the AtXUT1 gene resulted in shorter root hairs while the aerial part of wild-type and mutant were indistinguishable indicating that this type of acidic XyG structure plays a role specifically in root hair development [63]. MUR3, XLT2, XUT1 and the XSTs all act to transfer glycosyl-moieties to the same O-2 position of the XyG xylosyl-residue and are part of the same subclade of GT47. Hence, it is likely that the proteins responsible for the addition of other glycosyl groups to this position, including the arabinopyranosyland xylosyl- of D and U sidechains, respectively, will also be identified from this GT47 subclade.

Figure 4. Schematic representation of different types of XyG and known proteins involved in its synthesis and modification. Detailed linkage information of XyG structures can be found in Figure 1. At, Arabidopsis; Os, Oryza sativa; Tm, Tropaeolum majus; Pr, Pinus radiata; Eg, Eucalyptus grandis; Sl, Solanum lycopersicum. GT, glycosyltransferase; GH, glycoside hydrolase. Arrows and scissors indicate the site of glycosyltransferase or glycosyl hydrolase activity, respectively.
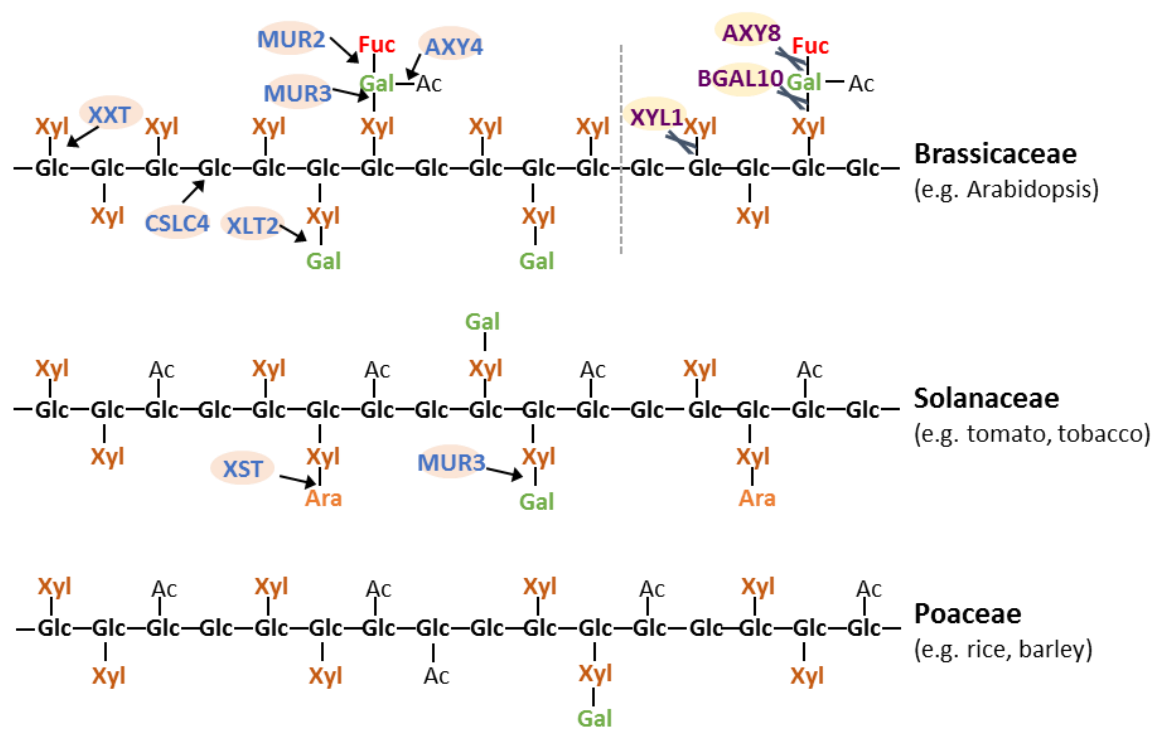

\begin{tabular}{|c|c|c|c|c|c|}
\hline Activity & Gene Name & Species & CAZy Family & Evidence & References \\
\hline Glucan synthase & CSLC4 & $A t, T m$ & GT2 & in vitro activity & {$[80]$} \\
\hline Xylosyltransferase & XXT1-5 & $A t, O s, P r$ & GT34 & loss of function, in vitro activity & {$[14,81-87]$} \\
\hline Galactosyltransferase & MUR3 & $A t, E g, S I, T m$ & GT47 & loss of function, in vitro activity & {$[69,88-90]$} \\
\hline Galactosyltransferase & $\mathrm{XLT} 2$ & $A t, T m$ & GT47 & loss of function & {$[69,89]$} \\
\hline Arabinosyltransferase & XST1, XST2 & $S I$ & GT47 & gain of function & [69] \\
\hline Galacturonsyltransferase & XUT1 & At & GT47 & loss of function & [63] \\
\hline Fucosyltransferase & MUR2/FUT1 & At & GT37 & loss of function, in vitro activity & {$[91,92]$} \\
\hline Acetyltransferase & AXY4, AXY4L & At & - & loss of function & [93] \\
\hline Xylosidase & AXY3/XYL1 & At & $\mathrm{GH} 31$ & loss of function, in vitro activity & {$[94,95]$} \\
\hline Fucosidase & AXY8 & At & GH95 & loss of function, in vitro activity & [75] \\
\hline Galactosidase & BGAL10 & At & GH35 & loss of function, in vitro activity & {$[96]$} \\
\hline
\end{tabular}


In Arabidopsis, one of the galactosyl-residues can be fucosylated by the $\alpha$-(1,2)-fucosyltransferase Fucosyltransferase1 (FUT1), a member of GT family 37 [91,92]. Genetic disruption of the gene encoding FUT1 in the mur2 mutant results in non-fucosylated XyG [92]. Despite this alteration in structure, mur2 plants display a normal growth habit and wall strength, although ultrastructural analysis revealed an alteration in trichome structure [92]. When the abundance of the donor substrate of FUT1, GDP-fucose, is reduced by genetic alteration of the nucleotide sugar conversion pathway in the murl mutant, the fucosylation of XyG is nearly abolished and a L-galactosyl residue is added to the galactosyl-moiety instead of the fucosyl-substituent to form a $\mathbf{J}$ sidechain $[92,97,98]$. In this case, the availability of donor substrate rather than the identity of the glycosyltransferase determines the sidechain present. FUT1 also exhibits promiscuity for the acceptor substrate as it can utilize a galacturonosyl group to produce the $\mathrm{Z}$ sidechain [63]. Based on the similarity in structure, it appears likely that FUT1 or another GT37 enzyme is involved in producing E sidechains found in some ferns and lycophytes. Despite the tolerance for alternative donor and acceptor residues, $\mathrm{XyG}$ fucosylation is regio-specific and typically occurs only on the last xylosyl-residue of the XyG oligosaccharide as in the XyG oligosaccharide motif XLFG, but not on the middle galactosyl-position. An XFFG unit has been found in the XyG of suspension cultured cells of sycamore (Acer pseudoplatanus) [99], but this structure has not been observed in a plant tissue and it is unclear if MUR2 or another fucosyltransferase is responsible for this transfer.

The galactosyl-residue can also be $O$-acetylated in Arabidopsis. A putative XyG $O$-acetyltransferase has been identified as Altered Xyloglucan 4 (AXY4), a protein which belongs to the Trichome Birefrigence-like (TBL) family [93]. It is not clear onto which position of the galactosyl-residue the acetyl group is initially transferred. AXY4 is required for XyG acetylation in leaves whereas a related TBL gene, Altered Xyloglucan 4-like ( $A X Y 4 L)$, is responsible for XyG acetylation in seeds. Absence of the $O$-acetyl substituent on $\mathrm{XyG}$ in the various tissues was not reported to affect tissue growth or morphology. In fact, a naturally occurring ecotype of Arabidopsis, Ty-0, found in the Scottish highlands, has a defect in AXY4 and a decrease in XyG acetylation, suggesting that XyG acetylation is at least partially dispensable for Arabidopsis in this particular environment [93].

$\mathrm{XyG}$ is synthesized in the Golgi and exported to the apoplast via exocytosis where it can undergo further metabolism, turn-over and/or maturation $[13,100]$. Known XyG modifying enzymes and hydrolases include XyG endotransglucosylase/endohydrolases (XTHs), and glycosidases such as the xylosidase Altered Xyloglucan 3 (AXY3)/Xylosidase 1 (XYL1), the fucosidase Altered Xyloglucan 8 (AXY8), and the galactosidase Beta-galactosidase 10 (BGAL10) [75,94-96,101-105]. XTHs are capable of cleaving and reforming the backbone glycosidic bonds of XyG polymers in rapidly growing plant tissues [104,105]. It is thought that this class of enzymes might be responsible for incorporating nascent $\mathrm{XyG}$ polymers into the existing cellulose/XyG network. While these enzyme activities have been found in the apoplast [106,107], it is possible that they are also present within the endomembrane system during polysaccharide biosynthesis and transport. $\mathrm{XyG}$ isolated from Arabidopsis axy 8 microsomes was found to have a higher degree of fucosylation than that of wild type plants, suggesting AXY8 is active on XyG both in the apoplast and in the endomembrane system [75]. Knocking out the xylosidase XYL1/AXY3 increased the overall abundance of XyG xylosylation in its corresponding Arabidopsis mutant, while the abundance of galactosylated XyG structures was increased in the bgal10 mutant. Both glycosidases seem to impact plant development as siliques are dwarfed in these mutants [94-96]. The Arabidopsis axy 8 mutant displays an increased abundance of fucosylated $\mathrm{XyG}$ oligosaccharides, but 
unexpectedly results in $\mathrm{XyG}$ that contains unusual $\mathrm{XyG}$ oligosaccharides such as XFG, GFGXXXG, and GFGXXFG [75]. When both the xylosidase and fucosidase were knocked-out in the axy 8 axy3 double mutant, the XyG structure consists of a 1:1 ratio of the XyG oligosaccharide motifs XXXG and XXFG in contrast to XLFG, XXFG, XXLG, XLXG and XXXG motifs usually found in Arabidopsis tissues. This data provides evidence that apoplastic glycosidases also play an important role in structurally diversifying XyG oligosaccharide motifs found in the wall [75].

\section{Outlook}

Initially, $\mathrm{XyG}$ research was focused on the description of $\mathrm{XyG}$ structure, thereby leading to the discovery of sidechain diversity and the distinct ordering of the sidechains that make up various XyG oligosaccharide motifs. While more than 20 different sidechain structures have been identified to date, there remain many plant families and entire orders for which the XyG structure has not been reported (Figures 2 and 3, Table S1) suggesting that further diversity may yet be discovered. Additional challenges remain to understand the order in which the oligosaccharide motifs are linked together and their functional significance. There is evidence that $\mathrm{XyG}$ consists of covalently-linked polysaccharide domains that bind to cellulose microfibrils interlaced with domains that span between adjacent microfibrils $[15,108]$. Different quantities of various XyG oligosaccharide motifs have been observed between these domains [7], however drawing functional conclusions is difficult and the overall patterning of the oligosaccharide motifs is still unclear.

With the advent of genetics and genomics many of the genes involved in XyG biosynthesis and metabolism have now been identified and characterized. This knowledge, together with the corresponding plant mutants, allows XyG structure to be altered in planta giving crucial insights into the function of XyG structural features in plant growth and development. While still in its infancy, a closer phylogenetic and protein structural investigation of the GTs involved in XyG biosynthesis will allow us to better understand the molecular basis for GT donor and acceptor specificity as well as the evolutionary history of the corresponding genes. The availability of powerful methods for analyzing XyG structure as well as the ability of plants to tolerate aberrant $\mathrm{XyG}$ structures make it uniquely suited as a model plant polysaccharide, which can be used to gain insight into the biosynthesis, deposition, metabolism and evolution of non-cellulosic polysaccharides in the plant cell wall.

\section{Acknowledgments}

We would like to mention the funding sources that supported the authors. Lifeng Liu and Lei Zhu were supported by a grant from the Energy Biosciences Institute; Alex Schultink by the Dickinson Chair for wood science and technology for Markus Pauly.

\section{Author Contributions}

Alex Schultink, Lifeng Liu, Lei Zhu and Markus Pauly wrote the paper.

\section{Conflicts of Interest}

The authors declare no conflict of interest. 


\section{References}

1. Carpita, N.C.; Gibeaut, D.M. Structural models of primary-cell walls in flowering plants - Consistency of molecular-structure with the physical-properties of the walls during growth. Plant J. 1993, 3, $1-30$.

2. Somerville, C.; Bauer, S.; Brininstool, G.; Facette, M.; Hamann, T.; Milne, J.; Osborne, E.; Paredez, A.; Persson, S.; Raab, T.; et al. Toward a systems approach to understanding plant-cell walls. Science 2004, 306, 2206-2211.

3. Kooiman, P. The constitution of tamarindus-amyloid. Recl. Trav. Chim. Pay. B 1961, 80, 849-865.

4. Keegstra, K.; Talmadge, K.W.; Bauer, W.; Albersheim, P. The structure of plant cell walls III. A model of the walls of suspension-cultured sycamore cells based on the interconnections of the macromolecular components. Plant Physiol. 1973, 51, 188-197.

5. Albersheim, P.; Darvill, A.; Roberts, K.; Sederoff, R.; Staehelin, A. Plant Cell Walls: From Chemistry to Biology; Garland Science, Taylor and Francis Group, LLC: New York, NY, USA, 2011; pp. 1-430.

6. Hayashi, T. Xyloglucans in the primary-cell wall. Annu. Rev. Plant Phys. 1989, 40, 139-168.

7. Pauly, M.; Albersheim, P.; Darvill, A.; York, W.S. Molecular domains of the cellulose/xyloglucan network in the cell walls of higher plants. Plant J. 1999, 20, 629-639.

8. McCann, M.; Roberts, K. Architecture of the primary cell wall. In The Cytoskeletal Basis of Plant Growth and Form; Academic Press: Waltham, MA, USA, 1991; pp. 109-129.

9. Thompson, D.S. How do cell walls regulate plant growth? J. Exp. Bot. 2005, 56, 2275-2285.

10. Anderson, C.T.; Carroll, A.; Akhmetova, L.; Somerville, C. Real-time imaging of cellulose reorientation during cell wall expansion in Arabidopsis roots. Plant Physiol. 2010, 152, 787-796.

11. Talbott, L.D.; Ray, P.M. Molecular size and separability features of pea cell wall polysaccharides Implications for models of primary wall structure. Plant Physiol. 1992, 98, 357-368.

12. Ha, M.-A.; Apperley, D.C.; Jarvis, M.C. Molecular rigidity in dry and hydrated onion cell walls. Plant Physiol. 1997, 115, 593-598.

13. Takeda, T.; Furuta, Y.; Awano, T.; Mizuno, K.; Mitsuishi, Y.; Hayashi, T. Suppression and acceleration of cell elongation by integration of xyloglucans in pea stem segments. Proc. Natl. Acad. Sci. USA 2002, 99, 9055-9060.

14. Cavalier, D.M.; Lerouxel, O.; Neumetzler, L.; Yamauchi, K.; Reinecke, A.; Freshour, G.; Zabotina, O.A.; Hahn, M.G.; Burgert, I.; Pauly, M.; et al. Disrupting two Arabidopsis thaliana xylosyltransferase genes results in plants deficient in xyloglucan, a major primary cell wall component. Plant Cell 2008, 20, 1519-1537.

15. Park, Y.B.; Cosgrove, D.J. A revised architecture of primary cell walls based on biomechanical changes induced by substrate-specific endoglucanases. Plant Physiol. 2012, 158, 1933-1943.

16. Meier, H.; Reid, J. Reserve polysaccharides other than starch in higher plants. In Plant Carbohydrates I; Springer: Heidelberg, Germany, 1982; pp. 418-471.

17. Rao, P.; Srivastava, H. Tamarind. In Industrial Gums, 2nd ed.; Academic Press: New York, NY, USA, 1973; pp. 369-411. 
18. Edwards, M.; Bowman, Y.; Dea, I.; Reid, J. A $\beta$-D-Galactosidase from nasturtium (Tropaeolum majus L.) cotyledons. Purification, properties, and demonstration that xyloglucan is the natural substrate. J. Biol. Chem. 1988, 263, 4333-4337.

19. Mishra, A.; Malhotra, A.V. Tamarind xyloglucan: A polysaccharide with versatile application potential. J. Mater. Chem. 2009, 19, 8528-8536.

20. Mishra, M.; Khandare, J. Evaluation of tamarind seed polysaccharide as a biodegradable carrier for colon specific drug delivery. Int. J. Pharm. Pharm. Sci. 2011, 3, 139-142.

21. Rolando, M.; Valente, C. Establishing the tolerability and performance of tamarind seed polysaccharide (TSP) in treating dry eye syndrome: Results of a clinical study. BMC Ophthalmol. 2007, 7, 5 .

22. Ghelardi, E.; Tavanti, A.; Celandroni, F.; Lupetti, A.; Blandizzi, C.; Boldrini, E.; Campa, M.; Senesi, S. Effect of a novel mucoadhesive polysaccharide obtained from tamarind seeds on the intraocular penetration of gentamicin and ofloxacin in rabbits. J. Antimicrob. Chemother. 2000, 46, 831-834.

23. Greffe, L.; Bessueille, L.; Bulone, V.; Brumer, H. Synthesis, preliminary characterization, and application of novel surfactants from highly branched xyloglucan oligosaccharides. Glycobiology 2005, 15, 437-445.

24. Brumer, H., 3rd; Zhou, Q.; Baumann, M.J.; Carlsson, K.; Teeri, T.T. Activation of crystalline cellulose surfaces through the chemoenzymatic modification of xyloglucan. J. Am. Chem. Soc. 2004, 126, 5715-5721.

25. Scheller, H.V.; Ulvskov, P. Hemicelluloses. Annu. Rev. Plant Biol. 2010, 61, 263-289.

26. Pauly, M.; Gille, S.; Liu, L.; Mansoori, N.; de Souza, A.; Schultink, A.; Xiong, G. Hemicellulose biosynthesis. Planta 2013, 238, 627-642.

27. Fry, S.C.; York, W.S.; Albersheim, P.; Darvill, A.; Hayashi, T.; Joseleau, J.P.; Kato, Y.; Lorences, E.P.; Maclachlan, G.A.; Mcneil, M.; et al. An unambiguous nomenclature for xyloglucan-derived oligosaccharides. Physiol. Plantarum 1993, 89, 1-3.

28. Tuomivaara, S.T.; Yaoi, K.; O’Neill, M.A.; York, W.S. Generation and structural validation of a library of diverse xyloglucan-derived oligosaccharides, including an update on xyloglucan nomenclature. Carbohydr. Res. 2014, doi:10.1016/j.carres.2014.06.031.

29. Kiefer, L.L.; York, W.S.; Darvill, A.G.; Albersheim, P. Xyloglucan isolated from suspensioncultured sycamore cell-walls is $O$-acetylated. Phytochemistry 1989, 28, 2105-2107.

30. York, W.S.; Oates, J.E.; van Halbeek, H.; Darvill, A.G.; Albersheim, P.; Tiller, P.R.; Dell, A. Location of the $O$-acetyl substituents on a nonasaccharide repeating unit of sycamore extracellular xyloglucan. Carbohydr. Res. 1988, 173, 113-132.

31. Sims, I.M.; Munro, S.L.; Currie, G.; Craik, D.; Bacic, A. Structural characterisation of xyloglucan secreted by suspension-cultured cells of Nicotiana plumbaginifolia. Carbohydr. Res. 1996, 293, $147-172$.

32. York, W.S.; Kolli, V.S.K.; Orlando, R.; Albersheim, P.; Darvill, A.G. The structures of arabinoxyloglucans produced by solanaceous plants. Carbohydr. Res. 1996, 285, 99-128.

33. Jia, Z.; Cash, M.; Darvill, A.G.; York, W.S. NMR characterization of endogenously $O$-acetylated oligosaccharides isolated from tomato (Lycopersicon esculentum) xyloglucan. Carbohydr. Res. 2005, 340, 1818-1825. 
34. Maruyama, K.; Goto, C.; Numata, M.; Suzuki, T.; Nakagawa, Y.; Hoshino, T.; Uchiyama, T. $O$-acetylated xyloglucan in extracellular polysaccharides from cell-suspension cultures of Mentha. Phytochemistry 1996, 41, 1309-1314.

35. Kamerling, J.P.; Schauer, R.; Shukla, A.K.; Stoll, S.; Halbeek, H.; Vliegenthart, J. Migration of $O$-acetyl groups in N,O-acetylneuraminic acids. Eur. J. Biochem. 1987, 162, 601-607.

36. Kabel, M.A.; de Waard, P.; Schols, H.A.; Voragen, A.G. Location of $O$-acetyl substituents in xylo-oligosaccharides obtained from hydrothermally treated Eucalyptus wood. Carbohydr. Res. 2003, 338, 69-77.

37. Pauly, M. Development of Analytical Tools to Study Plant Cell Wall Xyloglucan; Shaker Verlag GmbH: Herzogenrath, Germany, 1999; pp. 1-252.

38. Pauly, M.; Qin, Q.; Greene, H.; Albersheim, P.; Darvill, A.; York, W.S. Changes in the structure of xyloglucan during cell elongation. Planta 2001, 212, 842-850.

39. Peña, M.J.; Darvill, A.G.; Eberhard, S.; York, W.S.; O’Neill, M.A. Moss and liverwort xyloglucans contain galacturonic acid and are structurally distinct from the xyloglucans synthesized by hornworts and vascular plants. Glycobiology 2008, 18, 891-904.

40. Popper, Z.A.; Fry, S.C. Primary cell wall composition of bryophytes and charophytes. Ann. Bot. 2003, 91, 1-12.

41. Mikkelsen, M.D.; Harholt, J.; Ulvskov, P.; Johansen, I.E.; Fangel, J.U.; Doblin, M.S.; Bacic, A.; Willats, W.G. Evidence for land plant cell wall biosynthetic mechanisms in charophyte green algae. Ann. Bot. 2014, 114, 1217-1236.

42. Hoffman, M.; Jia, Z.; Pena, M.J.; Cash, M.; Harper, A.; Blackburn, A.R., 2nd; Darvill, A.; York, W.S. Structural analysis of xyloglucans in the primary cell walls of plants in the subclass Asteridae. Carbohydr. Res. 2005, 340, 1826-1840.

43. Lampugnani, E.R.; Moller, I.E.; Cassin, A.; Jones, D.F.; Koh, P.L.; Ratnayake, S.; Beahan, C.T.; Wilson, S.M.; Bacic, A.; Newbigin, E. In vitro grown pollen tubes of Nicotiana alata actively synthesise a fucosylated xyloglucan. PLoS One 2013, 8, e77140.

44. Hsieh, Y.S.; Harris, P.J. Structures of xyloglucans in primary cell walls of gymnosperms, monilophytes (ferns sensu lato) and lycophytes. Phytochemistry 2012, 79, 87-101.

45. Bremer, B.; Bremer, K.; Chase, M.; Fay, M.; Reveal, J.; Soltis, D.; Soltis, P.; Stevens, P. An update of the Angiosperm Phylogeny Group classification for the orders and families of flowering plants: APG III. Bot. J. Linn. Soc. 2009, 161, 105-121.

46. Kakegawa, K.; Edashige, Y.; Ishii, T. Xyloglucan from xylem-differentiating zones of Cryptomeria japonica. Phytochemistry 1998, 47, 767-771.

47. Hsieh, Y.S.; Harris, P.J. Xyloglucans of monocotyledons have diverse structures. Mol. Plant 2009, 2, 943-965.

48. O’Neill, M.A.; York, W.S. The composition and structure of plant primary cell walls. In The Plant Cell Wall, Blackwell Publishing: Oxford, UK, 2003; pp. 1-54.

49. Gibeaut, D.M.; Pauly, M.; Bacic, A.; Fincher, G.B. Changes in cell wall polysaccharides in developing barley (Hordeum vulgare) coleoptiles. Planta 2005, 221, 729-738.

50. Buckeridge, M.S. Seed cell wall storage polysaccharides: Models to understand cell wall biosynthesis and degradation. Plant Physiol. 2010, 154, 1017-1023. 
51. Vinueza, N.R.; Gallardo, V.A.; Klimek, J.F.; Carpita, N.C.; Kenttämaa, H.I. Analysis of xyloglucans by ambient chloride attachment ionization tandem mass spectrometry. Carbohydr. Polym. 2013, 98, 1203-1213.

52. Huisman, M.; Weel, K.; Schols, H.; Voragen, A. Xyloglucan from soybean (Glycine max) meal is composed of XXXG-type building units. Carbohydr. Polym. 2000, 42, 185-191.

53. Alonso-Simón, A.; Neumetzler, L.; García-Angulo, P.; Encina, A.; Acebes, J.; Álvarez, J.; Hayashi, T. Plasticity of xyloglucan composition in bean (Phaseolus vulgaris)-cultured cells during habituation and dehabituation to lethal concentrations of dichlobenil. Mol. Plant 2010, 3, 603-609.

54. Ren, Y.; Picout, D.R.; Ellis, P.R.; Ross-Murphy, S.B.; Reid, J. A novel xyloglucan from seeds of Afzelia africana Se. Pers.-Extraction, characterization, and conformational properties. Carbohydr. Res. 2005, 340, 997-1005.

55. Watt, D.; Brasch, D.; Larsen, D.; Melton, L. Isolation, characterisation, and NMR study of xyloglucan from enzymatically depectinised and non-depectinised apple pomace. Carbohydr. Polym. 1999, 39, 165-180.

56. Galvez-Lopez, D.; Laurens, F.; Quemener, B.; Lahaye, M. Variability of cell wall polysaccharides composition and hemicellulose enzymatic profile in an apple progeny. Int. J. Biol. Macromol. 2011, 49, 1104-1109.

57. Lahaye, M.; Falourd, X.; Quemener, B.; Ralet, M.C.; Howad, W.; Dirlewanger, E.; Arús, P. Cell wall polysaccharide chemistry of peach genotypes with contrasted textures and other fruit traits. J. Agric. Food Chem. 2012, 60, 6594-6605.

58. Ray, S.; Vigouroux, J.; Quémener, B.; Bonnin, E.; Lahaye, M. Novel and diverse fine structures in LiCl-DMSO extracted apple hemicelluloses. Carbohydr. Polym. 2014, 108, 46-57.

59. York, W.S.; Impallomeni, G.; Hisamatsu, M.; Albersheim, P.; Darvill, A.G. Eleven newly characterized xyloglucan oligoglycosyl alditols: The specific effects of sidechain structure and location on ${ }^{1} \mathrm{H}-\mathrm{NMR}$ chemical shifts. Carbohydr. Res. 1995, 267, 79-104.

60. Louvet, R.; Rayon, C.; Domon, J.-M.; Rusterucci, C.; Fournet, F.; Leaustic, A.; Crépeau, M.-J.; Ralet, M.-C.; Rihouey, C.; Bardor, M. Major changes in the cell wall during silique development in Arabidopsis thaliana. Phytochemistry 2011, 72, 59-67.

61. Lerouxel, O.; Choo, T.S.; Seveno, M.; Usadel, B.; Faye, L.; Lerouge, P.; Pauly, M. Rapid structural phenotyping of plant cell wall mutants by enzymatic oligosaccharide fingerprinting. Plant Physiol. 2002, 130, 1754-1763.

62. Crombie, H.J.; Chengappa, S.; Hellyer, A.; Reid, J.S. A xyloglucan oligosaccharide-active, transglycosylating $\beta$-D-glucosidase from the cotyledons of nasturtium (Tropaeolum majus L.) seedlings-Purification, properties and characterization of a cDNA clone. Plant J. 1998, 15, 27-38.

63. Peña, M.J.; Kong, Y.; York, W.S.; O’Neill, M.A. A galacturonic acid-containing xyloglucan is involved in Arabidopsis root hair tip growth. Plant Cell 2012, 24, 4511-4524.

64. Mishra, A.; Joshi, M.; Jha, B. Oligosaccharide mass profiling of nutritionally important Salicornia brachiata, an extreme halophyte. Carbohydr. Polym. 2013, 92, 1942-1945.

65. Hilz, H.; de Jong, L.E.; Kabel, M.A.; Verhoef, R.; Schols, H.A.; Voragen, A.G. Bilberry xyloglucan-Novel building blocks containing $\beta$-xylose within a complex structure. Carbohydr. Res. 2007, 342, 170-181. 
66. Aboughe-Angone, S.; Nguema-Ona, E.; Ghosh, P.; Lerouge, P.; Ishii, T.; Ray, B.; Driouich, A. Cell wall carbohydrates from fruit pulp of Argania spinosa: Structural analysis of pectin and xyloglucan polysaccharides. Carbohydr. Res. 2008, 343, 67-72.

67. Assor, C.; Quemener, B.; Vigouroux, J.; Lahaye, M. Fractionation and structural characterization of LiCl-DMSO soluble hemicelluloses from tomato. Carbohydr. Polym. 2013, 94, 46-55.

68. Jia, Z.; Qin, Q.; Darvill, A.G.; York, W.S. Structure of the xyloglucan produced by suspensioncultured tomato cells. Carbohydr. Res. 2003, 338, 1197-1208.

69. Schultink, A.; Cheng, K.; Park, Y.B.; Cosgrove, D.J.; Pauly, M. The identification of two arabinosyltransferases from tomato reveals functional equivalency of xyloglucan side chain substituents. Plant Physiol. 2013, 163, 86-94.

70. Hantus, S.; Pauly, M.; Darvill, A.G.; Albersheim, P.; York, W.S. Structural characterization of novel L-galactose-containing oligosaccharide subunits of jojoba seed xyloglucans. Carbohydr. Res. 1997, 304, 11-20.

71. Selvendran, R.R.; Ryden, P. Isolation and analysis of plant cell walls. In Methods in Plant Biochemistry; Dey, P.M., Ed.; Academic Press: New York, NY, USA, 1990; pp. 549-579.

72. Pauly, M.; Andersen, L.N.; Kauppinen, S.; Kofod, L.V.; York, W.S.; Albersheim, P.; Darvill, A. A xyloglucan-specific endo- $\beta$-1,4-glucanase from Aspergillus aculeatus: Expression cloning in yeast, purification and characterization of the recombinant enzyme. Glycobiology 1999, 9, 93-100.

73. Obel, N.; Erben, V.; Schwarz, T.; Kühnel, S.; Fodor, A.; Pauly, M. Microanalysis of plant cell wall polysaccharides. Mol. Plant 2009, 2, 922-932.

74. Watanabe, T.; Shida, M.; Murayama, T.; Furuyama, Y.; Nakajima, T.; Matsuda, K.; Kainuma, K. Xyloglucan in cell walls of rice hull. Carbohydr. Res. 1984, 129, 229-242.

75. Günl, M.; Neumetzler, L.; Kraemer, F.; de Souza, A.; Schultink, A.; Pena, M.; York, W.S.; Pauly, M. AXY8 encodes an $\alpha$-fucosidase, underscoring the importance of apoplastic metabolism on the fine structure of Arabidopsis cell wall polysaccharides. Plant Cell 2011, 23, 4025-4040.

76. Marcus, S.E.; Verhertbruggen, Y.; Herve, C.; Ordaz-Ortiz, J.J.; Farkas, V.; Pedersen, H.L.; Willats, W.G.T.; Knox, J.P. Pectic homogalacturonan masks abundant sets of xyloglucan epitopes in plant cell walls. BMC Plant Biol. 2008, 8, e60.

77. Brennan, M.; Harris, P.J. Distribution of fucosylated xyloglucans among the walls of different cell types in monocotyledons determined by immunofluorescence microscopy. Mol. Plant 2011, 4, $144-156$.

78. Lerouxel, O.; Cavalier, D.M.; Liepman, A.H.; Keegstra, K. Biosynthesis of plant cell wall polysaccharides-A complex process. Curr. Opin. Plant Biol. 2006, 9, 621-630.

79. Scheible, W.-R.; Pauly, M. Glycosyltransferases and cell wall biosynthesis: Novel players and insights. Curr. Opin. Plant Biol. 2004, 7, 285-295.

80. Cocuron, J.C.; Lerouxel, O.; Drakakaki, G.; Alonso, A.P.; Liepman, A.H.; Keegstra, K.; Raikhel, N.; Wilkerson, C.G. A gene from the cellulose synthase-like $C$ family encodes a $\beta-1,4$ glucan synthase. Proc. Natl. Acad. Sci. USA 2007, 104, 8550-8555.

81. Faik, A.; Price, N.J.; Raikhel, N.V.; Keegstra, K. An Arabidopsis gene encoding an $\alpha$-xylosyltransferase involved in xyloglucan biosynthesis. Proc. Natl. Acad. Sci. USA 2002, 99, 7797-7802.

82. Cavalier, D.M.; Keegstra, K. Two xyloglucan xylosyltransferases catalyze the addition of multiple xylosyl residues to cellohexaose. J. Biol. Chem. 2006, 281, 34197-34207. 
83. Ade, C.P.; Bemm, F.; Dickson, J.M.; Walter, C.; Harris, P.J. Family 34 glycosyltransferase (GT34) genes and proteins in Pinus radiata (radiata pine) and Pinus taeda (loblolly pine). Plant J. 2014, 78, 305-318.

84. Zabotina, O.A.; van de Ven, W.T.G.; Freshour, G.; Drakakaki, G.; Cavalier, D.; Mouille, G.; Hahn, M.G.; Keegstra, K.; Raikhel, N.V. Arabidopsis XXT5 gene encodes a putative $\alpha$-1,6-xylosyltransferase that is involved in xyloglucan biosynthesis. Plant J. 2008, 56, 101-115.

85. Vuttipongchaikij, S.; Brocklehurst, D.; Steele-King, C.; Ashford, D.A.; Gomez, L.D.; McQueen-Mason, S.J. Arabidopsis GT34 family contains five xyloglucan $\alpha$-1,6-xylosyltransferases. New Phytol. 2012, 195, 585-595.

86. Zabotina, O.A.; Avci, U.; Cavalier, D.; Pattathil, S.; Chou, Y.H.; Eberhard, S.; Danhof, L.; Keegstra, K.; Hahn, M.G. Mutations in multiple XXT genes of Arabidopsis reveal the complexity of xyloglucan biosynthesis. Plant Physiol. 2012, 159, 1367-1384.

87. Wang, C.; Li, S.; Ng, S.; Zhang, B.; Zhou, Y.; Whelan, J.; Wu, P.; Shou, H. Mutation in xyloglucan 6-xylosytransferase results in abnormal root hair development in Oryza sativa. J. Exp. Bot. 2014, PMID: 24834920.

88. Madson, M.; Dunand, C.; Li, X.; Verma, R.; Vanzin, G.F.; Caplan, J.; Shoue, D.A.; Carpita, N.C.; Reiter, W.D. The MUR3 gene of Arabidopsis encodes a xyloglucan galactosyltransferase that is evolutionarily related to animal exostosins. Plant Cell 2003, 15, 1662-1670.

89. Jensen, J.K.; Schultink, A.; Keegstra, K.; Wilkerson, C.G.; Pauly, M. RNA-Seq Analysis of developing nasturtium seeds (Tropaeolum majus): Identification and characterization of an additional galactosyltransferase involved in xyloglucan biosynthesis. Mol. Plant 2012, 5, 984-992.

90. Lopes, F.J.F.; Pauly, M.; Brommonshenkel, S.H.; Lau, E.Y.; Diola, V.; Passos, J.L.; Loureiro, M.E. The EgMUR3 xyloglucan galactosyltransferase from Eucalyptus grandis complements the mur3 cell wall phenotype in Arabidopsis thaliana. Tree Genet. Genomes 2010, 6, 745-756.

91. Perrin, R.M.; DeRocher, A.E.; Bar-Peled, M.; Zeng, W.Q.; Norambuena, L.; Orellana, A.; Raikhel, N.V.; Keegstra, K. Xyloglucan fucosyltransferase, an enzyme involved in plant cell wall biosynthesis. Science 1999, 284, 1976-1979.

92. Vanzin, G.F.; Madson, M.; Carpita, N.C.; Raikhel, N.V.; Keegstra, K.; Reiter, W.D. The mur2 mutant of Arabidopsis thaliana lacks fucosylated xyloglucan because of a lesion in fucosyltransferase AtFUT1. Proc. Natl. Acad. Sci. USA 2002, 99, 3340-3345.

93. Gille, S.; de Souza, A.; Xiong, G.; Benz, M.; Cheng, K.; Schultink, A.; Reca, I.B.; Pauly, M. $O$-acetylation of Arabidopsis hemicellulose xyloglucan requires AXY4 or AXY4L, proteins with a TBL and DUF231 domain. Plant Cell 2011, 23, 4041-4053.

94. Günl, M.; Pauly, M. AXY3 encodes a $\alpha$-xylosidase that impacts the structure and accessibility of the hemicellulose xyloglucan in Arabidopsis plant cell walls. Planta 2011, 233, 707-719.

95. Sampedro, J.; Pardo, B.; Gianzo, C.; Guitian, E.; Revilla, G.; Zarra, I. Lack of $\alpha$-xylosidase activity in Arabidopsis alters xyloglucan composition and results in growth defects. Plant Physiol. 2010, 154, 1105-1115.

96. Sampedro, J.; Gianzo, C.; Iglesias, N.; Guitian, E.; Revilla, G.; Zarra, I. AtBGAL10 is the main xyloglucan beta-galactosidase in Arabidopsis, and its absence results in unusual xyloglucan subunits and growth defects. Plant Physiol. 2012, 158, 1146-1157. 
97. Zablackis, E.; York, W.S.; Pauly, M.; Hantus, S.; Reiter, W.D.; Chapple, C.C.; Albersheim, P.; Darvill, A. Substitution of L-fucose by L-galactose in cell walls of Arabidopsis mur1. Science 1996, 272, 1808-1810.

98. Pauly, M.; Eberhard, S.; Albersheim, P.; Darvill, A.; York, W.S. Effects of the murl mutation on xyloglucans produced by suspension-cultured Arabidopsis thaliana cells. Planta 2001, 214, 67-74.

99. Hisamatsu, M.; Impallomeni, G.; York, W.S.; Albersheim, P.; Darvill, A.G. A new undecasaccharide subunit of xyloglucans with two $\alpha$-L-fucosyl residues. Carbohydr. Res. 1991, $211,117-129$.

100. Nishitani, K. Construction and restructuring of the cellulose-xyloglucan framework in the apoplast as mediated by the xyloglucan-related protein family-A hypothetical scheme. J. Plant Res. 1998, $111,159-166$.

101. Tabuchi, A.; Mori, H.; Kamisaka, S.; Hoson, T. A new type of endo-xyloglucan transferase devoted to xyloglucan hydrolysis in the cell wall of azuki bean epicotyls. Plant Cell Physiol. 2001, 42, 154-161.

102. Nishitani, K.; Tominaga, R. Endoxyloglucan transferase, a novel class of glycosyltransferase that catalyzes transfer of a segment of xyloglucan molecule to another xyloglucan molecule. J. Biol. Chem. 1992, 267, 21058-21064.

103. Fry, S.C.; Smith, R.C.; Renwick, K.F.; Martin, D.J.; Hodge, S.K.; Matthews, K.J. Xyloglucan endotransglycosylase, a new wall-loosening enzyme-activity from plants. Biochem. J. 1992, 282, 821-828.

104. Rose, J.K.C.; Braam, J.; Fry, S.C.; Nishitani, K. The XTH family of enzymes involved in xyloglucan endotransglucosylation and endohydrolysis: Current perspectives and a new unifying nomenclature. Plant Cell Physiol. 2002, 43, 1421-1435.

105. Eklof, J.M.; Brumer, H. The XTH gene family: An update on enzyme structure, function, and phylogeny in xyloglucan remodeling. Plant Physiol. 2010, 153, 456-466.

106. Vissenberg, K.; Martinez-Vilchez, I.M.; Verbelen, J.-P.; Miller, J.G.; Fry, S.C. In vivo colocalization of xyloglucan endotransglycosylase activity and its donor substrate in the elongation zone of Arabidopsis roots. Plant Cell 2000, 12, 1229-1237.

107. Iglesias, N.; Abelenda, J.A.; Rodiño, M.; Sampedro, J.; Revilla, G.; Zarra, I. Apoplastic glycosidases active against xyloglucan oligosaccharides of Arabidopsis thaliana. Plant Cell Physiol. 2006, 47, 55-63.

108. Dick-Pérez, M.; Zhang, Y.; Hayes, J.; Salazar, A.; Zabotina, O.A.; Hong, M. Structure and interactions of plant cell-wall polysaccharides by two-and three-dimensional magic-angle-spinning solid-state NMR. Biochemistry 2011, 50, 989-1000.

(C) 2014 by the authors; licensee MDPI, Basel, Switzerland. This article is an open access article distributed under the terms and conditions of the Creative Commons Attribution license (http://creativecommons.org/licenses/by/4.0/). 